\title{
Testing Gravity with Pulsars in the SKA Era
}

\section{Lijing Shao*1, Ingrid H. Stairs ${ }^{2}$, John Antoniadis ${ }^{3}$, Adam T. Deller ${ }^{4}$, Paulo C. C. Freire $^{3}$, Jason W. T. Hessels ${ }^{4,5}$, Gemma H. Janssen ${ }^{4}$, Michael Kramer ${ }^{3,6}$, Jutta Kunz $^{7}$, Claus Lämmerzahl ${ }^{8}$, Volker Perlick ${ }^{8}$, Andrea Possenti ${ }^{9}$, Scott Ransom ${ }^{10}$, Benjamin W. Stappers ${ }^{6}$, Willem van Straten ${ }^{11}$}

${ }^{1}$ School of Physics, Peking University, Beijing 100871, China

${ }^{2}$ Department of Physics and Astronomy, University of British Columbia, 6224 Agricultural Rd., Vancouver, BC V6T 1Z1, Canada

${ }^{3}$ Max-Planck-Institut für Radioastronomie, Auf dem Hügel 69, D-53121 Bonn, Germany

${ }^{4}$ ASTRON, The Netherlands Institute for Radio Astronomy, 7990 AA Dwingeloo, The

Netherlands

${ }^{5}$ Anton Pannekoek Institute for Astronomy, University of Amsterdam, Science Park 904, 1098 XH Amsterdam, The Netherlands

${ }^{6}$ Jodrell Bank Centre for Astrophysics, The University of Manchester, M13 9PL, United Kingdom

${ }^{7}$ University of Oldenburg, Department of Physics, 26111 Oldenburg, Germany

${ }^{8}$ University of Bremen, ZARM, 28359 Bremen, Germany

${ }^{9}$ INAF-Osservatorio Astronomico di Cagliari, via della Scienza 5, 09047 Selargius (CA), Italy

${ }^{10}$ National Radio Astronomy Observatory, 520 Edgemont Road, Charlottesville, VA 22903, USA

${ }^{11}$ Centre for Astrophysics and Supercomputing and ARC Centre for All-Sky Astrophysics

(CAASTRO), Swinburne University of Technology, PO Box 218 Hawthorn, VIC 3122, Australia

E-mails: 1shao@pku.edu.cn (LS); stairs@astro.ubc.ca (IHS)

The Square Kilometre Array (SKA) will use pulsars to enable precise measurements of strong gravity effects in pulsar systems, which yield tests of gravitational theories that cannot be carried out anywhere else. The Galactic census of pulsars will discover dozens of relativistic pulsar systems, possibly including pulsar - black hole binaries which can be used to test the "cosmic censorship conjecture" and the "no-hair theorem". Also, the SKA's remarkable sensitivity will vastly improve the timing precision of millisecond pulsars, allowing probes of potential deviations from general relativity (GR). Aspects of gravitation to be explored include tests of strong equivalence principles, gravitational dipole radiation, extra field components of gravitation, gravitomagnetism, and spacetime symmetries.

Advancing Astrophysics with the Square Kilometre Array

June 8-13, 2014

Giardini Naxos, Italy

\footnotetext{
* Speaker.
} 


\section{Introduction}

In November 1915, Albert Einstein published the final paper that completed his theory of gravity and spacetime now known as general relativity (GR), changing our view of gravity and spacetime for ever. During its first fifty years, GR was considered a theoretical tour-de-force but one with meager observational evidence. Only in the 1960s did technology usher in the remarkable field of experimental gravity (Misner et al. 1973; Will 1993). Over the subsequent fifty years, GR has passed all experimental tests in laboratories, in the Solar System and in various stellar systems, particularly in binary pulsars (Will 2014). Astrophysical observations using radio bands have played one of the most important parts in the history of testing GR, with the ability to make precise measurements and probe into the vicinity of compact bodies, namely black holes (BHs) and neutron stars (NSs). Particularly, radio timing of binary pulsars has precisely probed the gravitational properties of NSs and, for the first time, tested the radiative properties of gravity, demonstrating that gravitational waves exist and that compact binaries lose energy from their emission at the rates predicted by GR (Hulse \& Taylor 1975; Taylor et al. 1979; Kramer et al. 2006; Weisberg et al. 2010; Freire et al. 2012b; Antoniadis et al. 2013). This confirmation and precise characterisation of the radiative properties of gravity is not only critical from the point of view of our understanding of fundamental physics and many aspects of astrophysics, but it will also open, via gravitational wave detectors, a whole new window on the Universe. The Square Kilometre Array (SKA), as the most powerful member of the next generation of radio telescopes, will allow much more precise radio timing of pulsars and will discover dozens of rare relativistic binary systems. For this reason it will play a unique role in many areas of experimental gravity.

Despite its success over the last century, GR still faces great challenges on the research frontiers of modern physics. First, it is known to be incomplete, since it fails at the centre of BHs. Second, as a non-renormalisable field theory, it appears to be incompatible with quantum principles. Reconciling gravity with quantum mechanics stands out as one of the great challenges of fundamental physics today. Furthermore, concepts like dark matter and dark energy pose additional challenges for our understanding of gravity, and modifications of gravity theories have been proposed to explain these phenomena (Capozziello \& de Laurentis 2011), like the Tensor-VectorScalar (TeVeS) theory, which was advanced to explain dark matter (Bekenstein 2004).

The important thing to us is that pulsar timing can be used to test some of these theories. Pulsar timing uses large radio telescopes to record the times of arrival (TOAs) of pulsed signals, produced by the rotation of the pulsar. These TOAs depend on the rotational and astrometric parameters of the pulsar, dispersion in the interstellar medium that the signals traverse, and the motion of the radio telescope in the Solar System. If the pulsar is in a binary, the TOAs also depend on the orbital dynamics of the binary, which are determined by the underlying gravitational theory (Weisberg \& Taylor 1981; Damour \& Taylor 1992; Edwards et al. 2006). Some theories of gravity, like a family of TeVeS-like theories introduced in Freire et al. (2012b), result in orbital dynamics that is sufficiently different from GR to be detected in the timing of some binary pulsars. Therefore, precise timing measurements of these binary pulsars can constrain, or altogether rule out, some of these gravity theories.

For example, in the Jordan-Fierz-Brans-Dicke theory (Jordan 1959; Fierz 1956; Brans \& Dicke 1961), gravity is mediated by a scalar field, $\varphi$, in addition to the canonical metric field, $g_{\mu \nu}$. The 

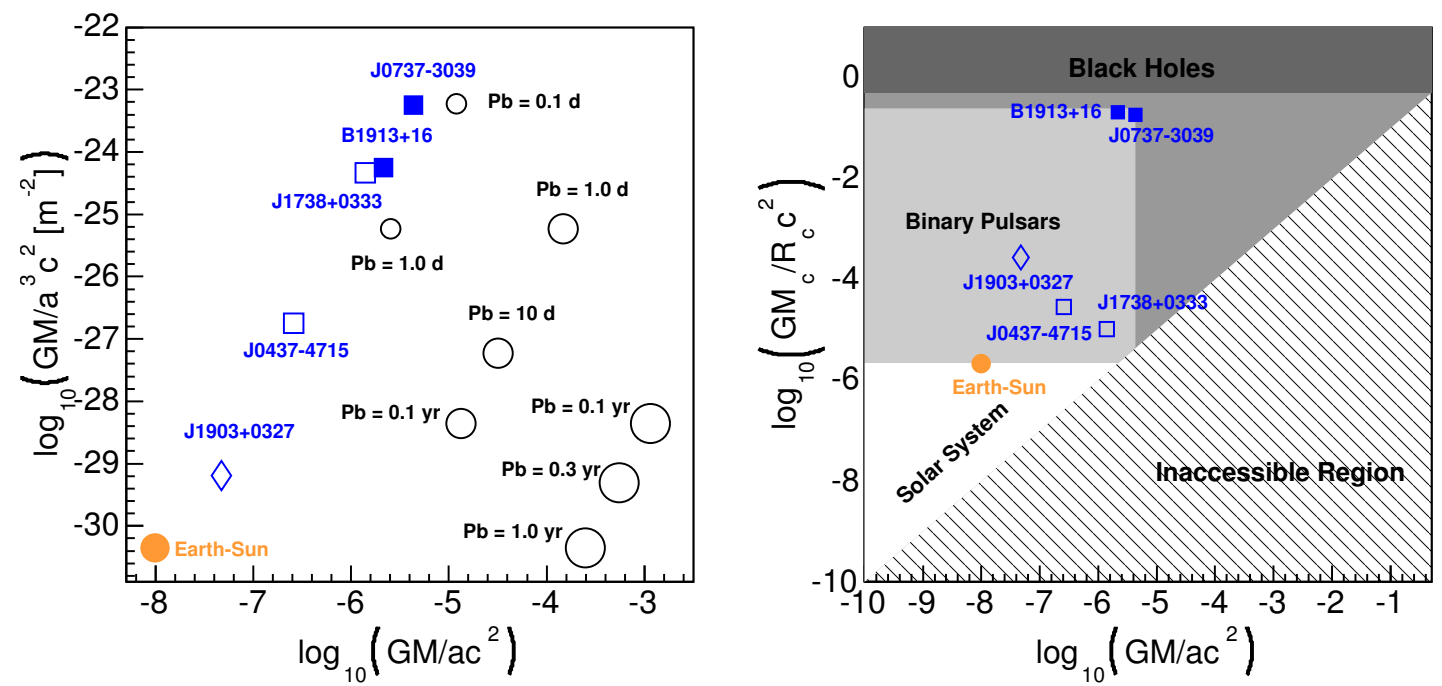

Figure 1: Left: Parameter space for quantifying the strength of a gravitational field (Psaltis 2008). The horizontal axis measures the gravitational potential and the vertical axis measures the spacetime curvature of an orbit with a semimajor axis, $a$, and a total mass, $M$ (here $G$ is the gravitational constant, and $c$ is the light speed). Solid and empty squares are known neutron star - neutron star (NS-NS) and neutron star - white dwarf (NS-WD) binaries, respectively. The diamond is a known neutron star - main sequence star binary. Circles from large to small are pulsar - Sgr A* BH, pulsar $-5000 M_{\odot} \mathrm{BH}$, and pulsar $-10 M_{\odot} \mathrm{BH}$ binaries with their assumed orbital periods aside. Right: The compactness of the gravitating companion star versus the compactness of the orbit; $M_{c}$ and $R_{c}$ are the mass and the radius of the companion star, respectively (Kramer et al. 2004).

variation of $\varphi$ in spacetime introduces observable effects in gravitational experiments, like the timevariation of the local gravitational constant $G$ and the existence of gravitational dipole radiation in a binary. In a more general class of scalar-tensor theories (Damour \& Esposito-Farèse 1993, 1996), the coupling strength of the scalar field $\varphi$ to matter depends on the field itself $\varphi$ as $\alpha_{0}+\varphi \beta_{0}$, where $\alpha_{0}$ and $\beta_{0}$ fully characterise the theory. Within some of the $\left(\alpha_{0}, \beta_{0}\right)$ space, non-perturbative effects, called "spontaneous scalarisation", may happen in compact bodies like NSs (Damour \& EspositoFarèse 1993), which can influence the orbital dynamics of a binary system dramatically. Similar effects are also possible within an extended family of TeVeS-like theories (Freire et al. 2012b). With their ability to probe strong-field effects, binary pulsars are ideal laboratories with which to study these alternative gravitational theories. With the non-detection of gravitational dipole radiation from radio pulsar timing, binary pulsars have already severely constrained the parameter spaces of these theories and provided the most stringent tests of the strong-field ${ }^{1}$ and radiative properties of gravity (Freire et al. 2012b; Antoniadis et al. 2013; Stairs 2003; Wex 2014).

\footnotetext{
${ }^{1}$ Compared to the coalescence of two compact objects (that will be the main source for ground-based gravitational wave detectors), two components of a binary pulsar are well separated, that might be prejudicially termed as "weak field". However, as shown by explicit calculations, "strong-field effects" associated with strongly self-gravitating NSs could have significant effects on the binary orbital dynamics (Damour \& Esposito-Farèse 1993).
} 


\subsection{The role of the Square Kilometre Array}

The Square Kilometre Array will have a major impact on radio astronomy. The SKA Phase 1 (SKA1) will feature roughly half of its total collecting area within a dense core, in the cases of SKA1-LOW and SKA1-MID. By synthesising a coherent sum of these elements, it will be possible to deeply search for new relativistic binary pulsars, over the whole sky, and to subsequently time them with great precision using the full SKA1-MID array (i.e. including the longer baselines). The SKA Phase 2 (SKA2) will provide another major leap in sensitivity, and thus also in timing precision. Both phases of the SKA will significantly advance experimental tests of gravitational theories to a new level (Kramer et al. 2004).

This will happen through two avenues. On the one hand, SKA1-MID and SKA2 will strongly improve the quality of the observations of the most interesting binaries currently known (e.g., PSR J0737-3039 (Burgay et al. 2003; Lyne et al. 2004; Kramer et al. 2006)) and triple systems (e.g., PSR J0337+1715 (Ransom et al. 2014)), in turn dramatically improving the quality of the existing tests and enabling proposed new tests. Compared with the current status quo, SKA1-MID will improve the timing precision for southern pulsars by about an order of magnitude, and SKA2 will improve it by up to two orders of magnitude. Different pulsars will gain different improvements in precision, depending on their sky locations, pulse characteristics, timing stabilities, and so on.

For precision pulsar timing, raw sensitivity is one of the most important factors. High cadence may also be needed for some experiments, in which case sub-arraying will be needed. Typically, TOAs for a particular pulsar are collected every few weeks, though in come cases a much higher cadence is necessary, e.g. to focus on orbital conjunction in order to measure the Shapiro delay. Sub-arraying can also help if many sources need to be timed, and if reduced sensitivity is acceptable. Though SKA1-MID will provide the highest-precision TOAs, regular measurements with SKA1-LOW may also be needed to track the time-variable interstellar propagation effects that also influence the TOAs. In all cases, coherently dedispersing the data is essential for achieving the maximum timing precision and accuracy. Such timing observations can already begin in an early phase of SKA1 (say, with 50\% of the collecting area available), though they will naturally obtain less precise TOAs than with the complete array. For some sources $50 \%$ sensitivity will be sufficient, while for others certain relativistic effects may only become clearly detectable once the full array is in place.

The SKA will also discover new relativistic systems with which to carry out similar and completely novel tests. With a larger field of view and high sensitivity, the SKA is more efficient in finding new pulsars. The larger sensitivity of the SKA means that a shorter integration time is needed to achieve the required signal to noise ratio. Consequently, in an "acceleration search" with an assumed constant orbital acceleration (Lorimer \& Kramer 2004), the smearing due to varying acceleration in an actual binary will be reduced, making the SKA much more effective than current telescopes at finding relativistic binaries. Simulations show that the SKA1 can discover a total of about 10,000 normal pulsars and perhaps as many as 1,800 millisecond pulsars (MSPs), with SKA1-LOW surveying the sky with the Galactic latitude $|b| \geq 5^{\circ}$, and SKA1-MID surveying the sky with the Galactic latitude $|b| \leq 10^{\circ}$ (Keane et al. 2015). The SKA2 will discover even more pulsars, and it is possible that among them there will be pulsar - black hole (PSR-BH) binaries 
(Kramer et al. 2004; Keane et al. 2015). The discovery of even a single tight PSR-BH system will open a new era of studying BH physics with great precision, including possible tests of the "cosmic censorship conjecture" and the "no-hair theorem" (Damour \& Esposito-Farèse 1998; Wex \& Kopeikin 1999; Kramer et al. 2004; Liu 2012; Liu et al. 2012). Figure 1 shows the vast unprobed regions in the gravity sector that can be probed with different kinds of PSR-BH systems.

\section{Relativistic Binaries}

Pulsar timing takes advantage of the tremendous rotational stability of pulsars, allowing us to treat them as free-falling clocks. As a pulsar is monitored over months and years, an ephemeris is calculated which accounts for every rotation of the pulsar and can be used to predict future pulse TOAs. A pulsar ephemeris always incorporates the spin frequency and generally at least one spin derivative accounting for rotational energy loss, plus astrometric parameters. Binary pulsar orbits require a further translation of reference frame, accomplished for most binary systems by the five Keplerian parameters describing an eccentric Keplerian orbit. In order to account for relativistic effects beyond a Keplerian orbit, a set of theory-independent "post-Keplerian" (PK) parameters are introduced (Damour \& Deruelle 1986; Damour \& Taylor 1992) (see Table 1 for an incomplete collection of the most important PK parameters). In practice, PK parameters could be contaminated by astrophysical effects other than relativistic effects due to gravity. For example, the observed orbital decay parameter, $\dot{P}_{b}$, has "kinematic" contributions, which result from the proper motion of the system and the difference of Galactic accelerations between that system and the Solar System Barycentre. Here unless otherwise stated, we assume that non-gravitational contributions are properly corrected or demonstrably negligible in effect. If so, in the case of two well-separated masses with negligible spin contributions, the PK parameters are functions of the well-measured Keplerian parameters, the component masses, the equation of state (EOS) of stellar matters, and the parameters describing the gravitational theory (Damour \& Taylor 1992) (in GR, the internal constitution of the star is irrelevant to a high "post-Newtonian" (PN) order, only the total masses matter - this is the "effacement" property to be discussed in section 3).

Measuring two PK parameters, we can use a specific theory of gravity to determine the component masses of the system; these are important to study stellar evolution theories and, in some cases, to constrain EOS (Watts et al. 2015). If more PK parameters are measured, then the theory can be tested by a self-consistent argument - using the masses derived in the first stage one should be able to predict the subsequent PK measurements. Another way of viewing this is the so-called "mass-mass diagram" (see Figure 2 for a GR-based example for PSR J0737-3039 (Kramer et al. 2006; Breton et al. 2008)). For a gravitational theory to pass the test(s), all curves in the diagram should intersect in some region, i.e., the theory must be able to describe the component masses in a self-consistent way (Taylor \& Weisberg 1982).

The current state-of-the-art binary in this area is the Double Pulsar (PSR J0737-3039A/B) (Burgay et al. 2003; Lyne et al. 2004), which enables five tests of GR. Figure 2 shows its massmass diagram, where seven curves corresponding to seven mass constraints intersect at one point (Kramer et al. 2006; Breton et al. 2008; Kramer \& Wex 2009), indicating that GR provides a selfconsistent description of all of its timing measurements. The Double Pulsar resides in the southern sky and will be an important target for the SKA. The timing precision of the SKA will enable tests 
Table 1: The most important PK parameters that could be used in pulsar timing of binaries (Damour \& Deruelle 1986; Damour \& Taylor 1992; Wex \& Kopeikin 1999; Lorimer \& Kramer 2004; Edwards et al. 2006). In practice, for a specific binary pulsar, only some PK parameters are measured, depending on the characteristics of the pulsar timing experiment.

\begin{tabular}{ll}
\hline \hline Parameter & \\
\hline$\dot{\omega}$ & time derivative of the longitude of periastron $\omega$ \\
$\gamma$ & amplitude of the Einstein delay \\
$\dot{P}_{b}$ & time derivative of the orbital period $P_{b}$ \\
$r$ & range of the Shapiro delay \\
$s$ & shape of the Shapiro delay \\
$\Omega_{\mathrm{SO}}$ & precession rate of the pulsar spin \\
$\delta_{\theta}$ & mismatch in eccentricities (see text) \\
$\dot{e}$ & time derivative of the orbital eccentricity $e$ \\
$\dot{x}$ & time derivative of the projected semimajor axis of the pulsar orbit $x$ \\
$\ddot{\omega}$ & second time derivative of the longitude of periastron \\
$\ddot{x}$ & second time derivative of the projected semimajor axis of the pulsar orbit \\
\hline
\end{tabular}

of relativistic effects beyond their first-order PN approximations (Kramer \& Wex 2009). Measuring higher-order PN effects is important since it allows the investigation of the effects from pulsar spin (Barker \& O’Connell 1975; Damour \& Schaefer 1988; Kramer \& Wex 2009). The fractional effects due to the next $\mathrm{PN}$ order $^{2}$ on the advance rate of periastron, namely $\dot{\omega}^{2 \mathrm{PN}} / \dot{\omega}^{1 \mathrm{PN}}$, and on the orbital decay, namely $\dot{P}_{b}^{3.5 \mathrm{PN}} / \dot{P}_{b}^{2.5 \mathrm{PN}}$, are both at the order of $\mathscr{O}\left(10^{-5}\right)$. Simulations showed that by obtaining a timing variance below $\sim 5 \mu$ s for the Double Pulsar we will reach the precision required to detect higher-order effects (Kramer \& Wex 2009); this is very likely with SKA1-MID, scaling from current observations. The SKA2 will have sub- $\mu$ s timing precision for the Double Pulsar (assuming its pulse profile shape can still be well modelled), raising the prospect of highly precise measurements of these higher-order parameters.

Besides the orbital dynamics of two point masses, spin contributions may play an important role in the observations of pulsar binaries (Barker \& O'Connell 1975). Due to the curvature of the spacetime produced by the companion star, the rotation axis of a freely falling object (here the pulsar) suffers a precession with respect to a distant observer; this effect is known as "geodetic precession". Because of it, over time different emitting regions of the magnetosphere will be visible from the Earth, thus causing a secular change in the observed pulse profile. This effect has been observed in some tight binary pulsars, for example, in PSRs B1913+16 (Weisberg et al. 1989; Kramer 1998), B1534+12 (Stairs et al. 2004; Fonseca et al. 2014), J1141-6545 (Manchester et al. 2010), J1906+0746 (Lorimer et al. 2006) and J0737-3039B (Perera et al. 2010). For PSRs B1534+12 and J0737-3039B we even have direct measurements of the precession rates, which match the GR predictions (Stairs et al. 2004; Fonseca et al. 2014; Breton et al. 2008). The SKA1-MID will have comparable sensitivity to the Arecibo telescope, meaning that significant improvement in pre-

\footnotetext{
${ }^{2}$ Effects due to the PN orders are those that are suppressed by the ratio of the orbital velocity, $v$, to the light speed, $c$, compared with those due to Newtonian gravity (Will 2014). It is denoted as " $n \mathrm{PN}$ " if the suppression factor is $\mathscr{O}\left(v^{2 n} / c^{2 n}\right)$.
} 


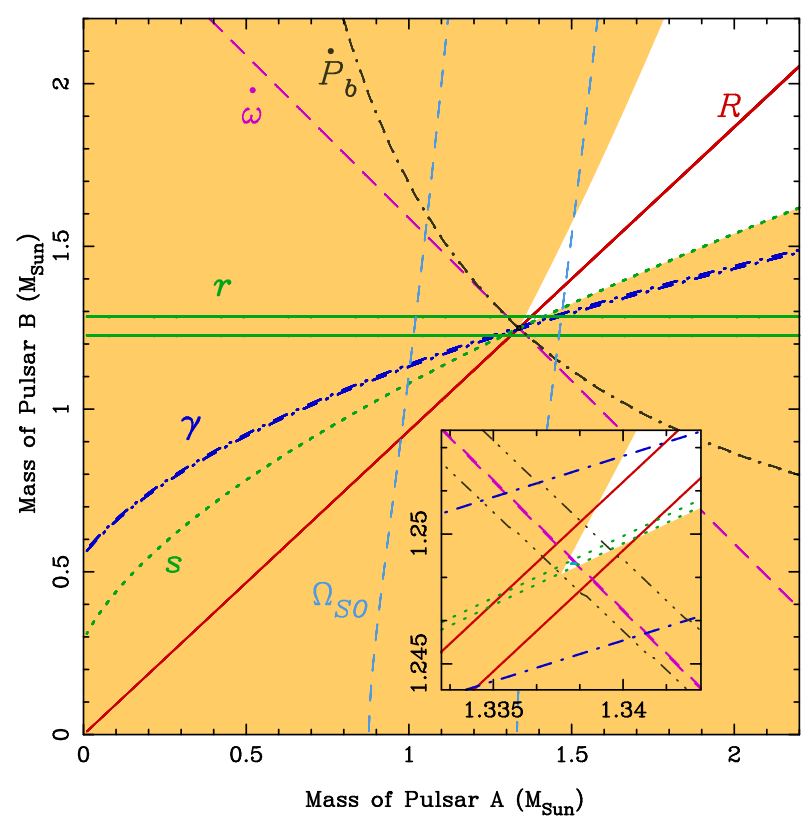

Figure 2: The mass-mass diagram of PSR J0737-3039A/B, also known as the Double Pulsar (Kramer et al. 2006; Breton et al. 2008) (Kramer et al., in prep.). In the figure the underlying gravitational theory is assumed to be GR. Shaded regions are forbidden by the individual mass functions because the sine of the orbital inclination must be $\leq 1$. The inset is an expanded view of the region of principal interest, where the intersection of seven curves at one point within measurement uncertainties proves the existence and uniqueness of a solution. For more details, see Kramer et al. (2006) and Breton et al. (2008).

cession measurements of the pulsars that are in the Arecibo sky will await the SKA2. However, precessing pulsars not visible to the Arecibo telescope will be prime targets for SKA1-MID. For example, in the Double Pulsar, the precession rate of the B pulsar can be measured by modelling the orientation of its magnetosphere as it causes "flickering" eclipses of pulsar A (Breton et al. 2008). The rapidly changing eclipse-period flux density will be much better measured by both phases of the SKA, leading to a more stringent test of the precession rate's agreement with GR.

Since in GR the total angular momentum must be conserved up to 2PN order, a change in the direction of the pulsar spin must be compensated by a change in the direction of the orbital angular momentum, contributing a variation to the projected semimajor axis of the orbit $(\dot{x} \neq 0)$. This spin-orbit coupling is also known as the Lense-Thirring effect or frame-dragging effect, and it is a small effect that is numerically at $2 \mathrm{PN}$ order for slowly rotating neutron stars, whose measurement requires a high timing precision (Damour \& Schaefer 1988). However, measuring it is important since it offers the potential to measure the moment of inertia of the pulsar (Damour \& Schaefer 1988; O'Connell 2004), of great interest for the EOS of cold dense nuclear matter (Watts et al. 2015).

For the Double Pulsar, the recycled pulsar (pulsar A) dominates the contribution to the LenseThirring precession, because it is a faster rotator. The effect on $\dot{x}$ will be almost impossible to 
measure, not only because in this case there is a close alignment of the orbital angular momentum and the spin of pulsar A (Ferdman et al. 2013), but also because the system is viewed almost edge-on (Kramer et al. 2006). However, the SKA Galactic census will discover many NS-NS systems (scaling from the current population, we expect 100 from the SKA1 and 180 from the SKA2 (Keane et al. 2015)), and there may well be binaries that will enable the direct measurement of $\dot{x}$. In order to achieve the goal, a tight binary with good timing precision is needed. In addition, the misalignment angle between the pulsar spin and the orbital norm should be reasonably large, i.e., geodetic precession should be observable. This requires a relatively large "supernova kick velocity" for the second-formed compact object — in other words, an iron-core-collapse supernova, instead of an electron-capture supernova as likely happened in the Double Pulsar (Ferdman et al. 2013). In such misaligned binaries, the frame dragging introduces nonlinear evolution in time in the longitude of periastron and its projected semimajor axis; thus one might be able to extract $\ddot{\omega}$ and $\ddot{x}$ from radio timing. This would impose useful constraints on the system's geometry (Wex \& Kopeikin 1999).

The Lense-Thirring effect also contributes to $\dot{\omega}$ with a magnitude comparable to that of $\dot{\omega}^{2 \mathrm{PN}}$ (Damour \& Schaefer 1988; Kramer \& Wex 2009). For the Double Pulsar, this can in principle be extracted from the advance rate of periastron. To do this we must determine the component masses very precisely using two other precise PK parameters. One of them (the shape of Shapiro delay, $s$ ) already possesses the required precision (Kramer et al. in prep.). For the other one, $\dot{P}_{b}$, the orbital decay due to the emission of GW waves, we will need to estimate the distance to the pulsar carefully, in order to estimate precisely the kinematic corrections to it. This can only be done with the timing precision provided by the SKA. Again, if this Lense-Thirring contribution to $\dot{\omega}$ can be estimated precisely, we will then be able to estimate the moment of inertia of pulsar A with similar precision. Because this measurement will be done for a pulsar with an exquisitely well-determined mass, it will be a very important constraint on the EOS for dense matter (Lattimer \& Schutz 2005; Kramer \& Wex 2009; Watts et al. 2015).

The SKA will also confirm general-relativistic orbital deformation for the first time. Damour $\&$ Deruelle (1986) used three different eccentricities to describe the timing of a pulsar. Two parameters were introduced in the pulsar timing formula, namely $\delta_{\theta}$ and $\delta_{r}$, where the former is in principle an observable (Damour \& Taylor 1992). However, it only adds a tiny periodic variance to the TOAs and the signal has a strong correlation with the Einstein delay that is caused by the transverse Doppler effect and the gravitational redshift, its amplitude is $e^{2} / \sqrt{1-e^{2}}$ in binaries of mass ratio near unity, where $e$ is the eccentricity of the orbit. Therefore, we will need highly eccentric orbits to "amplify" the effect, and highly relativistic orbits with large periastron advance rate to break the degeneracy with the Einstein delay. The SKA is likely to find such a system in the southern sky and time it with high precision. We simulate TOAs with orbital parameters similar to that of PSR B1913+16 (Hulse \& Taylor 1975; Weisberg et al. 2010) with the TEMPO software ${ }^{3}$. It is assumed that 60 TOAs with a precision $\sim 1 \mu \mathrm{s}$ are obtained per month with the SKA1. After 10 years of observation, the $\delta_{\theta}$ parameter can be determined to a precision $\sim 10 \%$. A more eccentric (or a more relativistic) binary can achieve a better test. These results support earlier simulations done with the Double Pulsar (Kramer \& Wex 2009).

\footnotetext{
${ }^{3} \mathrm{http} / / /$ tempo.sourceforge.net
} 
Where parameters in the timing model can be measured to high precision by other means, they can be restricted to an allowed range in the timing fit. This can lead to significantly reduced uncertainties for the remaining parameters in the presence of covariances. The most common source of external information is the estimation of position, proper motion and annual parallax using Very Long Baseline Interferometry (VLBI). The use of VLBI can improve gravitational tests when applied to pulsar binaries. For instance, astrometric information provided by VLBI was used to calculate the kinematic contamination of the measured orbital period derivative of the Double Pulsar, improving the potential accuracy of tests of gravitational radiation to the $0.01 \%$ level (Deller et al. 2009). In a second example, the previously unmeasured Shapiro delay for PSR J2222-0137 was precisely ascertained after the position, proper motion, and parallax were fixed using VLBI information (Deller et al. 2013). Since VLBI measures motions on the plane of the sky, it has different "blind spots" with respect to pulsar timing; VLBI has no "blind spots" to parallax at the ecliptic pole and proper motion in the ecliptic plane. With current VLBI instruments, precisions of 10 micro-arcseconds for parallax and 10 micro-arcseconds per year for proper motion can be reached (Deller et al. 2013). The SKA1 will allow a similar leap in VLBI precision as it will provide in the timing precision, and so VLBI using the SKA1 will be able to deliver better results for parallax and proper motion than timing in most cases for nearby pulsars (Paragi et al. 2015). These measurements will be very important to subtract the Shklovskii contribution (which usually dominates the error budget), and the differential Galactic rotation, and vertical acceleration in the Galactic potential (which also depend on relatively well-constrained Galactic models), thus measure the intrinsic orbital period change to high precision.

\section{Gravitational Dipole Radiation and Equivalence-Principle Tests}

One of the characteristics of GR is the property of "effacement" (Damour 1987). It states that the finite extents of two bodies in a binary are largely irrelevant to their orbital dynamics as long as they are well separated. Effacement is deeply rooted in the strong equivalence principle (SEP) (Will 1993). However, in alternative theories of gravity, the SEP is generally violated, and the strong gravitational fields associated with a NS may have measurable impact on the orbital dynamics of the binary and the trajectory of light propagation (Will 1993; Damour \& EspositoFarèse 1992, 1993). Figure 1 shows various binary systems, with the gravitational field strength characterised by the compactness and curvature of the orbit and the compactness of the companion star.

\subsection{Gravitational dipole radiation and time-variation of the gravitational constant}

A possible violation of the SEP could be identified by the occurrence of gravitational dipole radiation. The dipole radiation is at lower PN order than the canonical quadrupole radiation predicted by GR, thus in principle it can dominate over the quadrupole mode (Damour \& Esposito-Farèse 1992, 1993; Will 1993, 2014). It carries away the gravitational energy of the orbit more rapidly than that in GR, collapsing the binary more quickly, and modifying the waveform of gravitational radiation that can be detected by future ground-based GW detectors. The leading change in the orbital period of a binary is given by the dipolar contribution with a form $\dot{P}_{b}^{\text {dipole }} \propto\left(\alpha_{1}-\alpha_{2}\right)^{2}$, where $\alpha_{i}(i=1,2)$ is the effective coupling strength between body $i$ and the fields associated with 


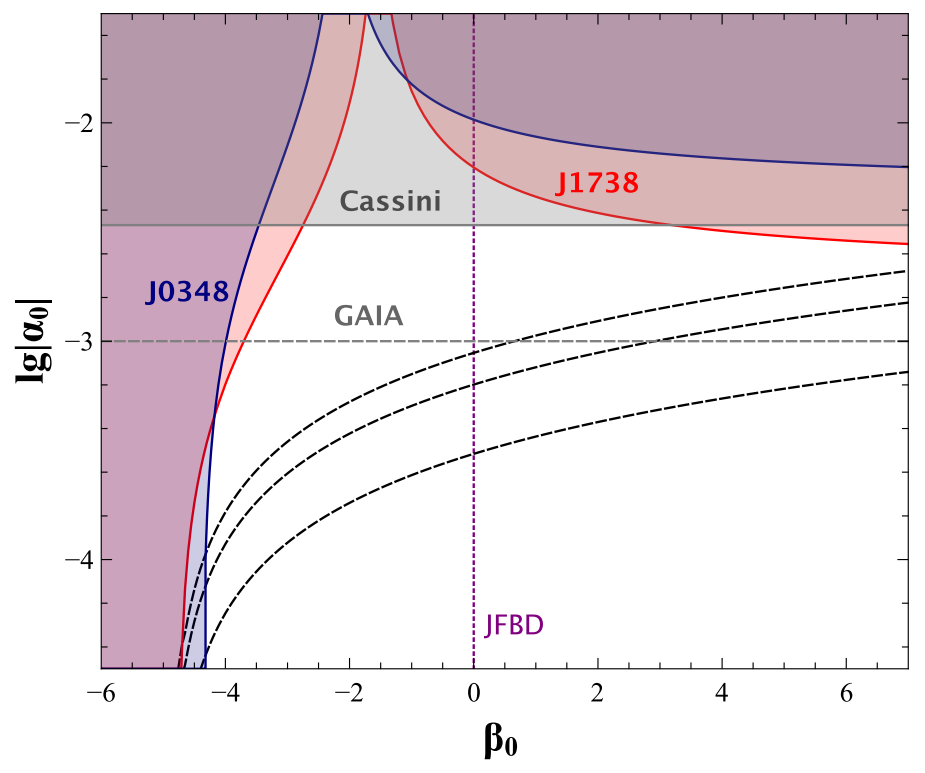

Figure 3: Constraints on the parameter space of scalar-tensor theories (figure courtesy of N. Wex). Coloured regions are excluded by current Solar System and pulsar experiments: Cassini spacecraft (Bertotti et al. 2003), PSR J1738+0333 (Freire et al. 2012b), and PSR J0348+0432 (Antoniadis et al. 2013). The EOS is chosen as MPA1. Black dashed lines are based on simulations for a MSP-BH system with $\left(M_{\mathrm{BH}}, M_{\mathrm{MSP}}\right)=$ $\left(10 M_{\odot}, 1.4 M_{\odot}\right), P_{b}=5 \mathrm{~d}$, and $e=0.8$; the constraints from top to bottom are based on: 10 years with a 100m class radio telescope, 5 years with the FAST telescope, and 5 years with the SKA (Liu et al. 2014). The vertical dashed purple line "JFBD" indicates the Jordan-Fierz-Brans-Dicke gravity. The horizontal dashed grey line "GAIA" indicates the limit expected from near-future Solar-System experiments, foremost from the astrometric satellite GAIA.

the dipole moment. In the best-studied gravitational theory of this type, the scalar-tensor theory (Damour \& Esposito-Farèse 1992; Will 1993), we generally have $\alpha_{\mathrm{NS}} \neq \alpha_{\mathrm{WD}}$ in a NS-WD binary (see the right panel of Figure 1). Therefore, NS-WD binaries are the ideal laboratories for testing these theories and, if they are correct, searching for the existence of gravitational dipole radiation, which if detected would falsify GR.

In these NS-WD binaries, the combined radio observation of the pulsar and optical observation of the WD can give rise to precise measurements of two component masses (Antoniadis et al. 2012, 2013; Wex 2014). The mass measurements can be used to predict the amount of gravitational radiation within a specific theory, and this in turn is confronted with the radio timing observation of the orbital decay caused by gravitational damping. As mentioned, the dipolar radiation can in principle be dominant over the quadrupolar radiation, therefore, agreement between the observed $\dot{P}_{b}$ and the quadrupole formula in GR stringently constrains the dipolar radiation contribution, as in the cases of PSRs J1738+0333 (Freire et al. 2012b) and J0348+0432 (Antoniadis et al. 2013).

In Figure 3, the constraints from those two binaries are plotted in the $\alpha_{0}-\beta_{0}$ parameter space mentioned in section 1. The best constraint from the Cassini spacecraft in the Solar System is also plotted. The limits from two binary pulsars stringently constrain the parameter space of scalartensor theories, especially when the $\beta_{0}$ parameter is negative. The reason for this is that, as showed by Damour \& Esposito-Farèse (1993), in this region NSs develop non-perturbative strong-field 
effects like "spontaneous scalarisation" that are absent in the weak-field experiments in the Solar System; such effects would cause a very large increase in the emission of dipolar gravitational waves. The more massive the pulsar the less negative $\beta_{0}$ has to be for the spontaneous scalarisation to appear. For this reason, the timing of PSR J0348+0432, a two-solar-mass NS in a relativistic orbit where the orbital decay has been measured with some precision, introduces uniquely stringent constraints on the allowed range of $\beta_{0}$ (Antoniadis et al. 2013).

In Jordan-Fierz-Brans-Dicke theory and (from a broader viewpoint) in the generic scalartensor theories, the scalar field plays the role of local gravitational constant, implying that the latter may vary with space and time (Damour \& Esposito-Farèse 1992; Will 1993). The possible time-variation in the gravitational constant contributes to the decay of a binary orbit (Damour et al. 1988; Wex 2014). The extra contribution can be constrained by the measurement of $\dot{P}_{b}$ for binary pulsars by assuming a null contribution from the dipole radiation. In general, however, a varying gravitational constant is accompanied by the dipole radiation (Lazaridis et al. 2009). With more than one binary pulsar, one can also conduct a joint constraint simultaneously on both the dipole radiation and the time-variation in the gravitational constant, utilising the fact that these two effects have different dependence on the orbital period of the binary (Lazaridis et al. 2009). The latest constraint from pulsars on the time-variation of the gravitational constant (Freire et al. 2012b), which relies on VLBI (Deller et al. 2008) and timing (Verbiest et al. 2008) of PSR J0437-4715 and J1738+0333, is already comparable to the best constraint from the Solar System experiments (Will 2014). Moreover, pulsar tests are sensitive to strong-field effects on $\dot{G}$ (Wex 2014).

These examples already show how important proper subtraction of the contributions from Galactic acceleration and the Shklovskii effect is, and how crucial VLBI is for reducing uncertainties in distances and proper motions. Furthermore, in the near future a more accurate understanding of the Galactic potential will also be available (e.g., by the GAIA satellite ${ }^{4}$ ), further improving the estimates of the kinematic corrections of $\dot{P}_{b}$.

The next generation of 30-m class optical telescopes, for examples, the Thirty Meter Telescope $(\mathrm{TMT})^{5}$, the Giant Magellan Telescope $(\mathrm{GMT})^{6}$, and the European Extremely Large Telescope (EELT $)^{7}$, may also significantly advance the optical observations of WDs, improving their mass and distance estimates; which will soon limit the precision of some of the current gravity tests (Freire et al. 2012b).

In short, in the SKA era, pulsar observations will improve significantly and maintain their unique importance in testing alternative theories of gravity, but will benefit extensively from inputting other techniques like VLBI and optical observation. They will be crucial for tests of some extended gravitational theories that incorporate dark matter and dark energy as gravitational effects.

If any relativistic PSR-BH binaries are discovered, they will also be promising test beds for gravitational dipolar radiation because the no-hair theorem also applies to scalar-tensor theories of gravity; this implies that $\mathrm{BHs}$ have no scalar charge ${ }^{8}$. A consequence of this is that any radiative

\footnotetext{
${ }^{4}$ http://sci.esa.int/gaia/

${ }^{5} \mathrm{http}: / /$ www.tmt.org/

${ }^{6}$ http://www.gmto.org/

${ }^{7}$ http://www.eelt.org.uk/

${ }^{8}$ However, see Herdeiro \& Radu (2014) for "Kerr BHs" with scalar hair after a complex, massive scalar field, minimally coupled to GR, is included (Herdeiro \& Radu 2014).
} 
test that constrains $\left(\alpha_{\mathrm{NS}}-\alpha_{\mathrm{BH}}\right)$ will therefore constrain $\alpha_{\mathrm{NS}}$ directly (Damour \& Esposito-Farèse 1998). The constraints from simulations of a hypothetical PSR-BH system $\left(M_{\mathrm{BH}}=10 \mathrm{M}_{\odot}, P_{b}=\right.$ 5 day and $e=0.8$ ) are depicted as dashed lines in Figure 3 (Liu et al. 2014). Five years of weekly observations with the SKA will constrain $\left|\alpha_{0}\right|$ down to the order of $\mathscr{O}\left(10^{-4}\right)$ (Liu 2012; Wex et al. 2013), improving upon the expected constraint from the GAIA satellite. Worthy to note that, unlike NS-WD binaries, these tests with PSR-BH binaries will also constrain the parameter space with $\beta_{0} \sim-1$.

\subsection{Equivalence-principle violation and its effects on orbital dynamics}

A possible violation of the SEP has also been investigated by tracing the orbital dynamics of small-eccentricity NS-WD binaries (Damour \& Schaefer 1991). The differential free fall rates of the NS and the WD in the potential of the Milky Way will induce a characteristic time evolution of the orbital eccentricity vector (Damour \& Schaefer 1991). The SEP violation tends to polarise the Laplace-Runge-Lenz vector of the orbit towards the direction of the Galactic acceleration. By using statistical studies on the eccentricity distribution of NS-WD binaries with a probabilistic assumption (Wex 2000), the dimensionless SEP violation parameter (the Nordtvedt parameter), $\eta$, was constrained to a comparable magnitude to that from the tests in the Solar System (Stairs et al. 2005; Gonzalez et al. 2011; Will 2014). A direct test of SEP with binary pulsars that uses the time-variations in orbital parameters was also developed (Freire et al. 2012a). It does not rely on statistical or probabilistic assumptions.

The recently discovered triple system, PSR J0337+1715 (Ransom et al. 2014), provides an opportunity to improve the SEP test by many orders of magnitude (Freire et al. 2012a). In this system, an outer WD orbits an inner NS-WD binary in less than one year. Usually, in the tests of SEP violation, the Galactic acceleration exerted on the binary is of the order $\mathscr{O}\left(10^{-10} \mathrm{~m} \mathrm{~s}^{-2}\right)$, which is the force that would "polarise" the inner orbit in the case of SEP violation. In PSR J0337+1715, the outer WD provides a "polarising" acceleration that is more than one million times larger than the Galactic acceleration on the inner pair. As a rough estimate, we use the acceleration in Eq. (4) of Damour \& Schaefer (1991), by replacing the relativistic rate of advance of periastron for the inner binary with the orbital angular velocity of the outer orbit (also see Eq. (8.18) in Will (1993)). Based on the current measurement precision of the orbital eccentricities in the triple system, a possible upper limit of the SEP violation parameter is estimated, $|\Delta| \lesssim 4 \times 10^{-7}$, which is $10^{4}$ times better than the current pulsar constraint (N. Wex, private communication). Due to this pulsar's detectability with the Arecibo telescope, observations with SKA1-MID will mostly improve the SEP-violation test via the accumulation of more data ${ }^{9}$, but the SKA2 will improve the timing precision by a factor of ten, which should improve the test by a corresponding factor.

It was estimated in Ransom et al. (2014) that there could be $\lesssim 100$ such systems with MSPs residing in the Galaxy. Since the SKA will discover almost all visible pulsars in the Galaxy (Keane et al. 2015), it is likely that it will discover other triple systems like PSR J0337+1715, and it is possible that the SKA might even discover a triple system consisting of an inner NS-WD close binary and where the outer star is another NS. Such a system could be (again) many orders of

\footnotetext{
${ }^{9}$ In principle, SKA1-MID is better due to less radio frequency interference (RFI), and much wider bands than that of the Arecibo telescope.
} 
magnitude more sensitive to SEP violation effects than PSR J0337+1715. The reason is that the SEP violation effects are proportional to $\left(\alpha_{1}^{\text {inner }}-\alpha_{2}^{\text {inner }}\right) \cdot \alpha_{3}^{\text {outer }}$ (Freire et al. 2012a), where $\alpha_{1,2}^{\text {inner }}$ are the couplings of the inner bodies to the scalar field and $\alpha_{3}^{\text {outer }}$ is the coupling of the outer body to the scalar field. If the outer body is a WD, then $\alpha_{3}^{\text {outer }} \simeq \alpha_{0}$ ( $\alpha_{0}$ is coupling in the weak field), but if it is a NS, then $\alpha_{3}^{\text {outer }}$ could, according to some alternative theories of gravity, be many orders of magnitude larger than $\alpha_{0}$. For such theories, the predicted SEP violation effects would be roughly " $\alpha_{\mathrm{NS}} / \alpha_{0}$ " times larger than their prediction for PSR J0337+1715, therefore making the system much more sensitive to the SEP violation predicted in these theories. The non-detection of SEP violation in such a system would provide a stringent constraint on alternative theories of gravity that predict such SEP violation.

\subsection{Local Lorentz invariance and local position invariance of gravity}

Besides the universality of the free fall, the other two aspects of the Einstein equivalence principle (Will 2014), namely local Lorentz invariance (LLI) and local position invariance (LPI) of gravity, can also be tested with pulsar timing experiments. Scenarios with LLI violation have recently raised great interests in the gravitational community, for examples, in the Einstein-æther theory and the Hořava-Lifshitz theory. Two generic frameworks for testing deviations from GR are the parametrised post-Newtonian (PPN) formalism (Will 1993, 2014) and the standard-model extension (SME) (Bailey \& Kostelecký 2006), both of which contain parameters for LLI violation.

Generically, violation of LLI and LPI leads to modifications of the orbital dynamics of binary pulsars and the spin evolution of solitary pulsars in characteristic ways (Damour \& Esposito-Farèse 1992; Bailey \& Kostelecký 2006; Wex \& Kramer 2007; Shao \& Wex 2012; Shao et al. 2013; Shao 2014; Shao \& Wex 2013). The LLI-violating modifications of the orbital dynamics of a binary pulsar introduce time-variations in the orbital eccentricity and orbital inclination, plus an extra contribution to the periastron advance rate, thus resulting in changes in the PK parameters, $\dot{e}, \dot{x}$, and $\dot{\omega}$ (Damour \& Esposito-Farèse 1992; Bailey \& Kostelecký 2006; Shao \& Wex 2012; Shao 2014). The violation of LLI also leads to spin precession of a solitary pulsar with respect to a fixed direction ${ }^{10}$ (Shao et al. 2013; Shao 2014), while the violation of LPI leads to spin precession of a solitary pulsar around its "absolute" acceleration towards the Galactic centre (Shao \& Wex 2013). They both change our view on the emission region of a pulsar, and result in characteristic changes versus time in the pulse profiles. Interestingly, modifications of the orbital dynamics and spin evolution are related and controlled by one set of parameters in both PPN and SME frameworks. These modifications can be jointly constrained and cross-checked. The current best constraints on the LLI of gravity and LPI of gravity are from pulsar experiments (Stairs et al. 2005; Shao \& Wex 2012; Shao et al. 2013; Shao \& Wex 2013; Shao 2014). It was shown that these constraints are proportional to the timing precision of binary pulsars. With the SKA, tests will be improved dramatically with better sensitivities. Simulations show that, if the signal to noise ratio of pulse profiles improves by a factor of 10 with the SKA, then the limits on Lorentz-violating parameters improve the same factor over the current best ones with solely 10-year observations (say, from 2020 to 2030). The acquisition of one stable profile biweekly is assumed for two MSPs in Shao et al.

\footnotetext{
${ }^{10}$ If there exists a preferred frame, the fixed direction will be the direction of the "absolute" velocity of the binary with respect to this preferred frame.
} 


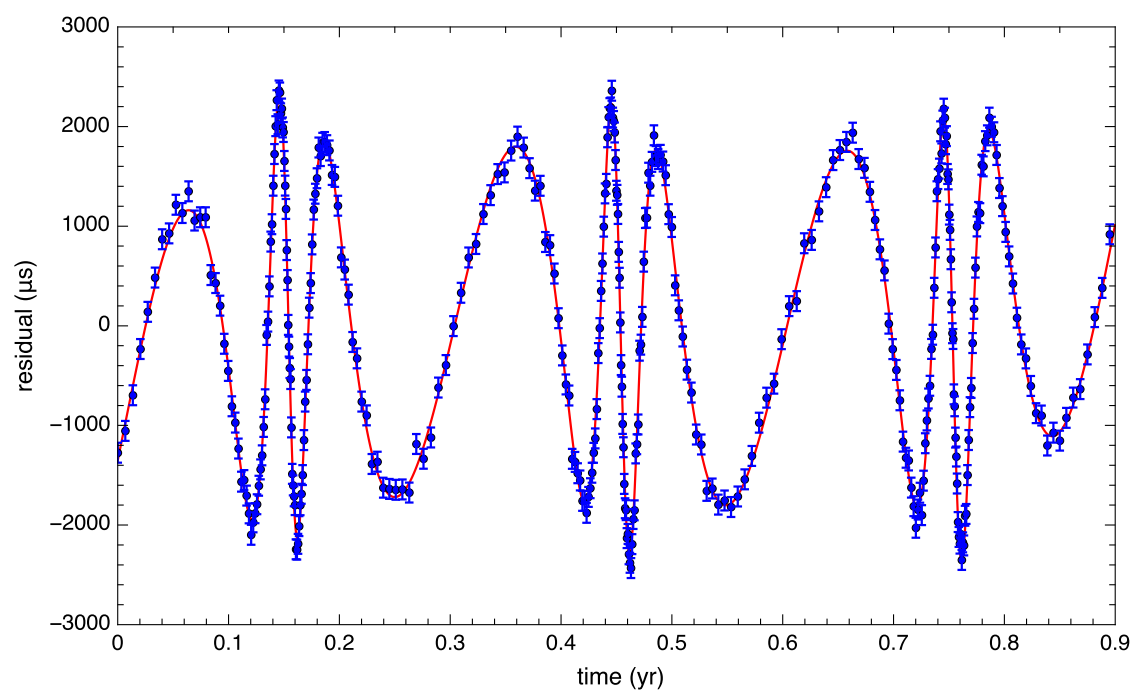

Figure 4: Characteristic pulsar timing residuals caused by the quadrupole moment of Sgr A* BH from simulated TOAs for three orbital phases (Liu et al. 2012) (figure courtesy of N. Wex). The orbital period is assumed to be $P_{b}=0.3 \mathrm{yr}$ and the eccentricity $e=0.5$. The red line is the fitted model.

(2013). If these SKA observations are properly combined with the 20-year pre-SKA observations (say, from 2000 to 2020), the improvement will be a factor of $\sim 50$. In addition, new stable MSPs from the Galactic census will be very helpful in breaking the degeneracies of parameters in the test, due to the vectorial and/or tensorial field condensations in the spacetime with such violations (Shao 2014).

\section{Pulsar - Black Hole Binaries}

BHs are predicted to be the ultimate outcome of massive stars in the theory of GR. Evidence for the existence of $\mathrm{BHs}$ in various astrophysical environments has accumulated during the past few decades, and is now rather robust (Narayan \& McClintock 2013). However, such observations are still indirect, and no high-precision tests of BHs are yet possible. Testing $\mathrm{BH}$ physics is a fantastic and intriguing goal in the fields of astronomy and physics. The goal of precision tests of properties of BHs will be achieved with the SKA (Kramer et al. 2004).

The combination of the sensitivity and the large field of view of the SKA will inevitably multiply the number of pulsars by tens to hundreds, making detection of rare objects like PSR-BH binaries possible. The shorter integration time in pulsar search (say, a 10-min integration as proposed) will be beneficial to discoveries of extremely relativistic binaries. A relativistic PSR-BH system in a clean environment represents a "holy grail" of pulsar astronomy. A vast unexplored parameter space in terms of gravitational potential, spacetime curvature and object compactness will then become accessible (see Figure 1). Pulsar - stellar-mass BH binaries, pulsar - intermediatemass $\mathrm{BH}$ binaries, and pulsars orbiting the $\mathrm{Sgr} \mathrm{A}^{*} \mathrm{BH}$ probe different new regimes of gravity where no other gravity experiments are yet able to be performed with precision tests (Wex \& Kopeikin 1999; Pfahl \& Loeb 2004). The pulsar - stellar-mass BH binary may naturally come from a primary binary composed of a $>20 M_{\odot}$ massive star and another $>8 M_{\odot}$ star, where the former ends 
up as a BH and the latter ends up as a NS. The pulsar - intermediate-mass BH binaries may exist in the centre of some globular clusters (Clausen et al. 2014; Hessels et al. 2015). Given the dense environment in the Galactic centre, there may be numerous NSs orbiting the Sgr A* BH, some of which may be detected as radio pulsars (Pfahl \& Loeb 2004). However, no pulsars close enough to Sgr A* BH for gravity tests have been detected yet. Recently, a magnetar was detected through X-ray (Mori et al. 2013; Kennea et al. 2013) and radio (Eatough et al. 2013) observations, however, it is not close enough to the Sgr A* BH for precision tests of gravity (Eatough et al. 2013, 2015). Because of scattering by the interstellar medium, in general we will need the SKA2 to detect these pulsars in the Galactic centre (Wharton et al. 2012; Wex et al. 2013; Eatough et al. 2015), although this will depend on which bands are included in SKA1-MID, for example, the inclusion of Band 5 will greatly enhance the prospects for doing this. For detecting the other two types of PSR-BH binaries, the SKA1 will start to contribute significantly.

In the case of relativistic PSR-BH binaries, the prominent effects from gravitation will, on one hand, reinforce better measurements of existing tests such as the Shapiro delay and the gravitational wave damping, and on the other hand, enable completely new tests of the "cosmic censorship conjecture" and the "no-hair theorem" (Damour \& Esposito-Farèse 1998; Wex \& Kopeikin 1999; Liu et al. 2012, 2014). The "cosmic censorship conjecture" states that for a realistic BH, there always exists an event horizon which prevents us from looking into its central singularity, thus preserving classical predictability in a spacetime. It imposes a maximum spin, $S_{\max }=G M^{2} / c$, on a rotating $\mathrm{BH}$ with a given mass $M$. The dimensionless spin of a $\mathrm{BH}, \chi \equiv c S / G M^{2}$, should always satisfy the constraint $\chi \leq 1$ (Misner et al. 1973). In alternative gravitational theories, this bound may be slightly violated, as in the Einstein-Gauss-Bonnet theory, where, besides the EinsteinHilbert term, the Gauss-Bonnet topological term is included in the Lagrangian of gravity (Kleihaus et al. 2011). The "no-hair theorem" states the uniqueness of a Kerr BH after it settles down. It relates the higher-order moments of a $\mathrm{BH}$ exclusively to its mass and spin. For example, if the "no-hair theorem" is valid, the dimensionless quadrupole of a BH, $q \equiv c^{4} Q / G^{2} M^{3}$ where $Q$ is the quadrupole moment of BH, should satisfy $q=-\chi^{2}$ (Misner et al. 1973). If a complex, massive scalar field, minimally coupled to GR, is introduced, then the "no-hair theorem" could be violated (Herdeiro \& Radu 2014).

For a relativistic PSR - Sgr A* BH system, the pulsar can be treated as a test particle in the first-order approximation (Hackmann \& Lämmerzahl 2008, 2012). The strategy of performing the tests of the "cosmic censorship conjecture" and the "no-hair theorem" was investigated in Liu et al. (2012). First of all, as usual in pulsar timing, we can estimate the mass of the Sgr A* BH through the measurement of the periastron advance rate. After that, a better mass determination and the geometry information can be obtained with the detection of the Shapiro delay and the Einstein delay. With the help of the frame-dragging effects (see section 2), the spin-orbit coupling then allows an initial extraction of an upper limit of the spin magnitude of the Sgr A* BH. Thus an initial test of the "cosmic censorship conjecture" becomes available by looking at the magnitude of $\chi$. This may enable us to look into the bizarre possibility of Sgr A* BH being a boson star (Kleihaus et al. 2012). With a better determination of the Sgr A* BH mass, the separation of the impact on the periastron advance from the $\mathrm{BH}$ spin is made plausible. This further determines the 3-dimensional spin of the BH with magnitude and direction. An extraction of the periodic effects of the quadrupole from timing residuals will then allow a quantitative test of the "no-hair theorem". 
The timing residual from the quadrupole of $\mathrm{Sgr} \mathrm{A} * \mathrm{BH}$ has a characteristic shape, as illustrated in Figure 4 for a hypothetical system with an orbital period $P_{b} \simeq 0.3 \mathrm{yr}$ and an eccentricity $e \simeq 0.5$ (Liu et al. 2012). According to simulations for an orbit with a period of several months, the spin and the quadrupole can be measured with precisions of $\sim 0.1 \%$ and $\sim 1 \%$ respectively after five years of observations with the SKA. Therefore, the test of the "no-hair theorem" can be performed at the $\sim 1 \%$ level by then (Liu et al. 2012).

Similarly, in the cases of stellar-mass BH and intermediate-mass BH binaries, the spin information of the $\mathrm{BH}$ can be extracted with the help of frame dragging as well if the orbit is tight enough (Wex \& Kopeikin 1999). For the stellar-mass BH companion, a relativistic binary can provide much better constraints on the scalar-tensor theories than the tests with gravitational waves from inspiralling binaries (Damour \& Esposito-Farèse 1998). These constraints will also be an order of magnitude better than the expected near-future constraint from the GAIA satellite when $\beta_{0}$ is negative or small; see Figure 3 for results from a simulated PSR-BH system (Liu et al. 2014).

\section{Conclusion and Outlook}

By the 2020s, radio astronomy will undergo a revolution brought by the onset of the SKA1. Its pulsar survey will deliver a large population of pulsars, among which there will be $\sim 1800$ MSPs. The most stable ones of them will be used to perform a number of precision gravity tests. The improvement in the sensitivity of the timing precision allows us a closer look at many aspects of the foundation of gravity. In the era of the SKA, the existing tests will be pushed to new extremes, and completely new tests will also be made possible with systems including NS-NS binaries, NSWD binaries and NS-BH binaries. Advances from the theoretical side will also contribute to the area of experimental gravity with novel tests. It is indeed a virtue of nature that pulsar binaries of different types are equipped with different characteristics to perform different tests with. For example, the NS-NS systems provide extremely precise tests of GR, in particular periodic, nondissipative effects in orbital dynamics (e.g. with the Einstein delay and general-relativistic orbital deformation), while the NS-WD and NS-BH systems are suitable to study the dissipative radiative property of gravity in the presence of asymmetry in gravitational binding energy (e.g. tests of the gravitational dipole radiation and the constancy of the gravitational constant). Furthermore, PSR$\mathrm{BH}$ binaries will be useful to test the $\mathrm{BH}$ physics (e.g. the "cosmic censorship conjecture" and the "no-hair" theorem). Tests of gravitational theories from many facets in the era of the SKA will profoundly advance our understandings of fundamental physics.

The output of gravity tests with pulsars has two possibilities. In the first possibility, GR is falsified. This will certainly provide an important hint for our further understanding of the fundamental interaction, gravity, and the nature of spacetime. In the second possibility, GR is confirmed to new precision with flying colours. This will further reinforce our confidence in using GR as a tool to investigate other phenomena, for example, using GR effects to make mass measurements of binary pulsars, helping to deepen our understandings of non-perturbative QCD effects (Watts et al. 2015). It also casts experimental constraints on constructing new gravitational theories in relevant areas, for example, to explain the nature of dark matter and dark energy. In both cases, tests of gravitational theories with great precision will provide important clues to reconcile GR and quantum principles. 
As a final remark, with the better timing capability and new discoveries of stable pulsars from the SKA, the pulsar timing array (PTA) projects will leap towards a new era as well. Complementary to the strong-field tests discussed here, PTAs make tests of gravitational theories with gravitational waves in the radiative regime available (Janssen et al. 2015).

\section{Acknowledgement}

We thank Norbert Wex for kindly providing figures and carefully reading the manuscript.

\section{References}

Antoniadis, J., Freire, P. C. C., Wex, N., et al. 2013, Science, 340, 448

Antoniadis, J., van Kerkwijk, M. H., Koester, D., et al. 2012, MNRAS, 423, 3316

Bailey, Q. G. \& Kostelecký, V. A. 2006, Phys. Rev. D, 74, 045001

Barker, B. M. \& O'Connell, R. F. 1975, Phys. Rev. D, 12, 329

Bekenstein, J. D. 2004, Phys. Rev. D, 70, 083509

Bertotti, B., Iess, L., \& Tortora, P. 2003, Nature, 425, 374

Brans, C. \& Dicke, R. H. 1961, Physical Review, 124, 925

Breton, R. P., Kaspi, V. M., Kramer, M., et al. 2008, Science, 321, 104

Burgay, M., D'Amico, N., Possenti, A., et al. 2003, Nature, 426, 531

Capozziello, S. \& de Laurentis, M. 2011, Phys. Rep., 509, 167

Clausen, D., Sigurdsson, S., \& Chernoff, D. F. 2014, MNRAS, 442, 207

Damour, T. 1987, The problem of motion in Newtonian and Einsteinian gravity., ed. S. W. Hawking \& W. Israel, 128-198

Damour, T. \& Deruelle, N. 1986, Ann. Inst. Henri Poincaré Phys. Théor., 44, 263

Damour, T. \& Esposito-Farèse, G. 1992, Classical and Quantum Gravity, 9, 2093

—. 1992, Phys. Rev. D, 46, 4128

—. 1993, Physical Review Letters, 70, 2220

—. 1996, Phys. Rev. D, 54, 1474

-. 1998, Phys. Rev. D, 58, 042001

Damour, T., Gibbons, G. W., \& Taylor, J. H. 1988, Physical Review Letters, 61, 1151

Damour, T. \& Schaefer, G. 1991, Physical Review Letters, 66, 2549

-. 1988, Nuovo Cimento B, 101, 127

Damour, T. \& Taylor, J. H. 1992, Phys. Rev. D, 45, 1840

Deller, A. T., Bailes, M., \& Tingay, S. J. 2009, Science, 323, 1327

Deller, A. T., Boyles, J., Lorimer, D. R., et al. 2013, ApJ, 770, 145

Deller, A. T., Verbiest, J. P. W., Tingay, S. J., \& Bailes, M. 2008, ApJL, 685, L67

Eatough, R. P., Falcke, H., Karuppusamy, R., et al. 2013, Nature, 501, 391

Eatough, R. P., Lazio, T. J. W., Casanellas, J., et al., 2015, “Observing Radio Pulsars in the Galactic Centre with the Square Kilometre Array", in proc. Advancing Astrophysics with the Square Kilometre Array, PoS(AASKA14)045

Edwards, R. T., Hobbs, G. B., \& Manchester, R. N. 2006, MNRAS, 372, 1549

Ferdman, R. D., Stairs, I. H., Kramer, M., et al. 2013, ApJ, 767, 85 
Fierz, M. 1956, Helv. Phys. Acta, 29, 128

Fonseca, E., Stairs, I. H., \& Thorsett, S. E. 2014, ApJ, 787, 82

Freire, P. C. C., Kramer, M., \& Wex, N. 2012a, Classical and Quantum Gravity, 29, 184007

Freire, P. C. C., Wex, N., Esposito-Farèse, G., et al. 2012b, MNRAS, 423, 3328

Gonzalez, M. E., Stairs, I. H., Ferdman, R. D., et al. 2011, ApJ, 743, 102

Hackmann, E. \& Lämmerzahl, C. 2008, Physical Review Letters, 100, 171101

—. 2012, Phys. Rev. D, 85, 044049

Herdeiro, C. A. R. \& Radu, E. 2014, Physical Review Letters, 112, 221101

Hessels, J. W. T., Possenti, A., Bailes, M., et al., 2015, "Pulsars in Globular Clusters with the SKA", in proc. Advancing Astrophysics with the Square Kilometre Array, PoS(AASKA14)047

Hulse, R. A. \& Taylor, J. H. 1975, ApJL, 195, L51

Janssen, G. H., Hobbs, G., McLaughlin, M., et al., 2015, "Gravitational wave astronomy with the SKA", in proc. Advancing Astrophysics with the Square Kilometre Array, PoS(AASKA14)037 Jordan, P. 1959, Zeitschrift fur Physik, 157, 112

Keane, E. F., Bhattacharyya, B., Kramer, M., et al., 2015, "A Cosmic Census of Radio Pulsars with the SKA", in proc. Advancing Astrophysics with the Square Kilometre Array, PoS(AASKA14)040

Kennea, J. A., Burrows, D. N., Kouveliotou, C., et al. 2013, ApJL, 770, L24

Kleihaus, B., Kunz, J., \& Radu, E. 2011, Physical Review Letters, 106, 151104

Kleihaus, B., Kunz, J., \& Schneider, S. 2012, Phys. Rev. D, 85, 024045

Kramer, M. 1998, ApJ, 509, 856

Kramer, M., Backer, D. C., Cordes, J. M., et al. 2004, New Astron. Rev., 48, 993

Kramer, M., Stairs, I. H., Manchester, R. N., et al. 2006, Science, 314, 97

Kramer, M. \& Wex, N. 2009, Classical and Quantum Gravity, 26, 073001

Lattimer, J. M. \& Schutz, B. F. 2005, ApJ, 629, 979

Lazaridis, K., Wex, N., Jessner, A., et al. 2009, MNRAS, 400, 805

Liu, K. 2012, PhD thesis (The University of Manchester)

Liu, K., Eatough, R. P., Wex, N., \& Kramer, M. 2014, MNRAS, 445, 3115

Liu, K., Wex, N., Kramer, M., Cordes, J. M., \& Lazio, T. J. W. 2012, ApJ, 747, 1

Lorimer, D. R. \& Kramer, M. 2004, Handbook of Pulsar Astronomy (Cambridge University Press)

Lorimer, D. R., Stairs, I. H., Freire, P. C. C., et al. 2006, ApJ, 640, 428

Lyne, A. G., Burgay, M., Kramer, M., et al. 2004, Science, 303, 1153

Manchester, R. N., Kramer, M., Stairs, I. H., et al. 2010, ApJ, 710, 1694

Misner, C. W., Thorne, K. S., \& Wheeler, J. A. 1973, Gravitation (San Francisco: W. H. Freeman and Company)

Mori, K., Gotthelf, E. V., Zhang, S., et al. 2013, ApJL, 770, L23

Narayan, R. \& McClintock, J. E. 2013, ArXiv e-prints, arXiv:1312.6698

O’Connell, R. F. 2004, Physical Review Letters, 93, 081103

Paragi, Z., Godfrey, L., Reynolds, C., et al., 2015, "Very Long Baseline Interferometry with the SKA", in proc. Advancing Astrophysics with the Square Kilometre Array, PoS(AASKA14)143

Perera, B. B. P., McLaughlin, M. A., Kramer, M., et al. 2010, ApJ, 721, 1193

Pfahl, E. \& Loeb, A. 2004, ApJ, 615, 253

Psaltis, D. 2008, Living Reviews in Relativity, 11, 9 
Ransom, S. M., Stairs, I. H., Archibald, A. M., et al. 2014, Nature, 505, 520

Shao, L. 2014, Physical Review Letters, 112, 111103

Shao, L., Caballero, R. N., Kramer, M., et al. 2013, Classical and Quantum Gravity, 30, 165019

Shao, L. \& Wex, N. 2012, Classical and Quantum Gravity, 29, 215018

-. 2013, Classical and Quantum Gravity, 30, 165020

Stairs, I. H. 2003, Living Reviews in Relativity, 6, 5

Stairs, I. H., Faulkner, A. J., Lyne, A. G., et al. 2005, ApJ, 632, 1060

Stairs, I. H., Thorsett, S. E., \& Arzoumanian, Z. 2004, Physical Review Letters, 93, 141101

Taylor, J. H., Fowler, L. A., \& McCulloch, P. M. 1979, Nature, 277, 437

Taylor, J. H. \& Weisberg, J. M. 1982, ApJ, 253, 908

Verbiest, J. P. W., Bailes, M., van Straten, W., et al. 2008, ApJ, 679, 675

Watts, A. L., Xu, R., Espinoza, C., et al., 2015, "Probing the neutron star interior and the Equation of State of cold dense matter with the SKA", in proc. Advancing Astrophysics with the Square Kilometre Array, PoS(AASKA14)043

Weisberg, J. M., Nice, D. J., \& Taylor, J. H. 2010, ApJ, 722, 1030

Weisberg, J. M., Romani, R. W., \& Taylor, J. H. 1989, ApJ, 347, 1030

Weisberg, J. M. \& Taylor, J. H. 1981, General Relativity and Gravitation, 13, 1

Wex, N. 2000, in Astronomical Society of the Pacific Conference Series, Vol. 202, IAU Colloq. 177: Pulsar Astronomy - 2000 and Beyond, ed. M. Kramer, N. Wex, \& R. Wielebinski, 113

—. 2014, ArXiv e-prints, arXiv:1402.5594

Wex, N. \& Kopeikin, S. M. 1999, ApJ, 514, 388

Wex, N. \& Kramer, M. 2007, MNRAS, 380, 455

Wex, N., Liu, K., Eatough, R. P., et al. 2013, in IAU Symposium, Vol. 291, IAU Symposium, ed. J. van Leeuwen, 171-176

Wharton, R. S., Chatterjee, S., Cordes, J. M., Deneva, J. S., \& Lazio, T. J. W. 2012, ApJ, 753, 108 Will, C. M. 1993, Theory and Experiment in Gravitational Physics (Cambridge University Press) —. 2014, Living Reviews in Relativity, 17, 4 